\title{
A commentary on "Apical suspension is underutilized for repair of stage IV pelvic organ prolapse: an analysis of national practice patterns in the United States"
}

\author{
Ghazaleh Rostaminia $^{1}$ (D) \\ Received: 20 May 2020 / Accepted: 1 June 2020 / Published online: 15 June 2020 \\ (C) The International Urogynecological Association 2020
}

This retrospective study utilized the American College of Surgeons National Surgical Quality Improvement Program database (NSQIP) to assess nationwide utilization of apical suspension procedures for the treatment of complete POP, which by definition involves descent of the vaginal apex. The authors aimed to describe practice patterns in surgical repair of complete uterovaginal prolapse and evaluate patient characteristics associated with various surgical approaches.

During 2006-2016, 2784 women with a mean age of $64.6 \pm 11.0$ years were identified. Only $51.9 \%$ of patients with stage IV POP underwent an apical suspension or obliterative procedure. Concurrent hysterectomy was performed in $47.5 \%(n=1332)$ of women, and vaginal hysterectomy was the most common surgical route $(70.2 \%$ of hysterectomies, $n=927$ ). Among women who underwent hysterectomy, only $38.6 \%(n=502)$ had an apical suspension or colpocleisis. Post-hysterectomy apical suspension was performed in $61.2 \%$ of patients. Based on a multivariable logistic regression analysis, the only factor associat- ed with an apical suspension was hysterectomy status (aOR 0.37, CI 0.32-0.44, $p<0.001$ ), adjusting for patient age, smoking status, obesity, and functional status. Complication rates were similar between surgeries with and without an apical suspension $(8.2 \%$ versus $7.0 \%$, $p=0.269$ ). The median operative time of surgeries that included an apical repair was 37 min longer $(p<0.001)$.

In summary, this study showed that only $\sim 52 \%$ of women who underwent surgery for stage IV vaginal prolapse received the appropriate surgical apical repair for this condition. Failure to perform an apical suspension is likely multifactorial, but lack of adequate training in these procedures, the perceived increased risk of complications associated with these procedures, and longer operative time, particularly in patients with higherrisk for surgery, may be the potential reasons. It appears that fellowship level training is necessary to attain a level of clinical competence to perform the proposed quality measures and more complicated surgical procedures when needed.

Ghazaleh Rostaminia

ghazalerostaminia@yahoo.com

1 NorthShore University HealthSystem, Skokie, IL, USA 